\title{
Lidil
}

Revue de linguistique et de didactique des langues

$45 \mid 2012$

Pratiques de formation à la lecture-écriture des adultes en parcours d'insertion

\section{Regards socio-didactiques sur les pratiques de formation linguistique pour les adultes en parcours d'insertion}

\section{Véronique Rivière}

\section{(2) OpenEdition}

\section{Journals}

Édition électronique

URL : http://journals.openedition.org/lidil/3215

DOI : $10.4000 /$ lidil.3215

ISSN : 1960-6052

\section{Éditeur}

UGA Éditions/Université Grenoble Alpes

Édition imprimée

Date de publication : 15 mai 2012

Pagination : 163-176

ISBN : 978-2-84310-226-4

ISSN : $1146-6480$

\section{Référence électronique}

Véronique Rivière, « Regards socio-didactiques sur les pratiques de formation linguistique pour les adultes en parcours d'insertion », Lidil [En ligne], 45 | 2012, mis en ligne le 15 novembre 2013, consulté le 30 avril 2019. URL : http://journals.openedition.org/lidil/3215 ; DOI : 10.4000/lidil.3215 


\title{
Regards socio-didactiques sur les pratiques de formation linguistique pour les adultes en parcours d'insertion
}

\author{
Véronique Rivière*
}

\begin{abstract}
RÉSUMÉ
Cet article final constitue une discussion de quelques-uns des thèmes principaux qui se dégagent du présent numéro : langue et compétence professionnelle, langue écrite et contextualisation didactique, langue écrite et réflexivité. Cette discussion porte sur quatre points. Il s'agit tout d'abord d'interroger la signification de la notion « langue comme compétence professionnelle ». Ensuite, nous centrons la discussion sur les relations de domination que les conditions socio-économiques et politiques, dans le champ de la formation linguistique pour les adultes en insertion sociale ou professionnelle, font peser sur la mise en place de dispositifs didactiques. Ensuite, nous mettons en évidence le rôle majeur que peuvent jouer les démarches de réflexion sur la langue dans ces situations spécifiques de formation d'adultes. Et enfin, nous caractérisons les pratiques de formation telles que décrites et analysées dans le numéro, comme des pratiques fortement transpositives et des pratiques étayantes.
\end{abstract}

\section{ABSTRACT}

This final article aims to discuss the main topics this review has tackled: literacy and working skills, literacy and didactic contextualisation, literacy and reflexivity. This discussion includes four topics. First, we bring up significations of "language as working skill". Secondly we focus on the factors that shape socio-economic and political domination in the field of linguistic training for adults and how they impact the didactic organisation. Next, we underscore the important role of reflexive approaches of language in these specific adult training situations. Finally, we characterize linguistic training pratices displayed in the review with a focus on transposition and scaffolding.

* Université Lumière - Lyon 2, UMR ICAR. 
Cette contribution conclusive, en guise de discussion finale et interprétative, propose de parcourir à rebours l'ensemble des articles du numéro sur les pratiques de formation à la langue écrite dans les parcours d'insertion des adultes, en suggérant quelques réflexions ou interrogations sur leurs enjeux didactiques, institutionnels, transversales aux différents articles et en repérant également des lignes de force qu'il me semble intéressant d'éclairer ici. Ce regard modestement rétrospectif se veut socio-didactique, c'est-à-dire que nous envisageons la réflexion sur un objet didactique, la langue et ses modes de transmission à visée d'insertion, dans ses intrications politiques, socioéconomiques, idéologiques et sociolinguistiques (Rispail et Blanchet, 2011). Ainsi nous tentons tout d'abord, de mettre en question le rapport, devenu évident dans le champ de la formation d'adultes à visée d'insertion, entre langue et compétence professionnelle. Ensuite, nous abordons la problématique ancienne en didactique des langues et du français de la contextualisation des pratiques didactiques à la lumière nouvelle de ce terrain de la formation d'adultes à visée d'insertion. Dans un troisième temps, nous pointons la pertinence de la réflexivité au sein des dispositifs d'appropriation. Enfin, nous discutons des pratiques de formation sur le plan méthodologique et sur le plan de leur caractérisation au sein de ce numéro de Lidil.

\section{La langue (écrite) comme compétence professionnelle?}

La formation linguistique de base se déroule de plus en plus au sein du secteur professionnel ou de la pré-professionnalisation pour les jeunes adultes ${ }^{1}$. En outre, depuis une décennie environ, les langues se situent au cœur des enjeux de professionnalisation, quels que soient les niveaux de qualification des acteurs au travail, aussi bien dans la sphère institutionnelle que politique ${ }^{2}$. En effet, les acteurs du monde politique et économique ont bien compris le rôle de contrôle de l'activité des opérateurs (Boutet, 2001) et de levier économique et d'efficacité au travail que pouvaient représenter la ou les langues de travail. Cela amène nombre

1. Dans le présent numéro, six des neuf contributions s'appuient sur des contextes de formation linguistique au sein du milieu professionnel ou à visée professionnelle.

2. À titre d'exemple, la réforme de la formation professionnelle de 2004 , les lois successives de politique migratoire instituant la langue française comme pré-requis indispensable pour l'accès à l'emploi. 
de spécialistes ou experts à assimiler les pratiques langagières (il s'agit du français ici surtout) à des compétences professionnelles. Et il est vrai que de nombreux travaux scientifiques ont très bien décrit les ressorts et les enjeux du langage au travail ${ }^{3}$ : enjeux d'efficacité productive, de compréhension du travail. Cependant, ils ont également mis en évidence que la langue au travail n'est pas seulement opérative mais revêt aussi enjeux symboliques, psycho-sociaux et expérientiels (Fraenkel et Borzeix, 2001). Pourtant, la locution « la langue comme compétence professionnelle », dont la formulation semble se généraliser depuis de la loi de 2004 sur la formation professionnelle et qu'on retrouve désormais sous la plume de certains didacticiens, pose un rapport d'équivalence ou d'assimilation entre langue (écrite) et compétence professionnelle et réduit la langue en situation de travail (et son apprentissage) à son utilisation strictement opérative. Cela mérite, à ce titre, d'être interrogé du point de vue socio-didactique. En effet, si on peut tout à fait accepter que, dans la situation de travail, l'activité professionnelle puisse être en partie ou exclusivement langagière ${ }^{4}$, dans la situation didactique, on peut se demander si les formateurs en langue forment à des compétences professionnelles et techniques ou à des ressources mobilisables dans le cadre de compétences professionnelles et techniques. En d'autres termes, comment, du point de vue didactique, les formateurs peuventils envisager ce rapport d'équivalence et mettre en place des dispositifs qui le servent? Nous faisons l'hypothèse que ce n'est pas complètement tenable (et pas souhaitable). Plus globalement, comment les dispositifs didactiques mis en place articulent-ils la visée d'efficacité du salarié à son poste de travail avec une visée de formation langagière plus holistique de la personne, nécessaire dans un processus d'insertion?

Le travail didactique à partir de supports pédagogiques écrits ou oraux peut permettre de coller au mieux aux situations de travail, mais H. Adami et V. André soulignent la possible complexité des écrits sur le plan sémiotique et discursif et leur difficile didactisation par les formateurs. De plus, la séparation, dans les référentiels, des compétences orales

3. Voir par exemple les travaux francophones du réseau Langage et Travail, de Filliettaz sur la formation professionnelle initiale, mais aussi le champ des Work place studies.

4. Voir la distinction désormais classique de Lacoste (1995) entre « langage sur le travail », « langage au travail », « langage comme travail ». Ajoutons que pour le travail du formateur ou de l'enseignant de langue, le langage est effectivement le cœur de l'activité. 
et écrites laisse de côté le caractère oralographique et plurisémiotique (Grossman, 1998) des situations de communication professionnelle. Une didactique des écrits au travail combinant analyse sémiologique des documents par corps de métier et conception de principes méthodologiques semble encore à constituer.

Les simulations, consistant à reproduire les situations de communication professionnelle dans la situation didactique peuvent également porter leurs fruits (Mourlhon-Dallies, 2008). Néanmoins, on sait que, même en faisant « comme si », les possibilités de transférer des compétences restent limitées parce que les situations réelles de travail sont « remplies » d'un univers de référence (le rythme de travail, les collègues, les objets, les machines sont présents et sont constitutifs bien souvent de l'activité de langage au travail) absent pendant la séance de formation. De même, comment sur le plan didactique, peut être pris en compte le caractère très indexical du langage par rapport aux objets techniques par exemple?

L'observation des situations de travail par les formateurs et leur traduction en objectifs ou contenus constitue une autre ressource permettant l'articulation entre situation de formation et situation de travail, mais on peut se demander si là est bien leur rôle. Et ont-ils les moyens d'aller observer les salariés de la propreté puis les salariés du bâtiment, puis de traduire les situations en contenus didactisables et en objectifs de formation linguistique? Il semble désormais acquis que le processus ingénierique complet (de la demande de formation à la réalisation de la formation) fasse partie des prérogatives du formateur. S'il a sans aucun doute à connaitre le contexte professionnel quotidien des salariés, pour autant, l'énorme tâche de transposition qu'il a à effectuer alourdit sa mission. F. Mourlhon-Dallies (2008: 82) résume très bien ce problème : " la démarche du FLP ${ }^{5}$ ne saurait se résumer à la transmutation directe d'analyses de la parole au travail en objectifs linguistiques de cours de langue, aussi solide que soit l'ingénierie de formation mise en œuvre ${ }^{6} »$. Rappelons à ce titre que le FLP n'est pas un ensemble de contenus linguistiques mais une démarche d'ingénierie s'appuyant sur une démarche scientifique d'analyse des discours professionnels.

5. FLP : Français langue professionnelle.

6. Ajoutons aussi que la logique marchande des formations soumises à appel d'offre a pour conséquence que les organismes de formation en concurrence ne mutualisent plus autant les ressources pédagogiques constituées par branche de métiers par exemple. 
Il semble donc qu'entre le formateur en langue à visée professionnelle ou aux savoirs de base et la situation de travail, il manque un maillon ingénierique.

\section{Langue (écrite) en formation de base : les dilemmes de la contextualisation}

Les référentiels de compétences nombreux, outre leur rôle de modélisation de l'activité professionnelle et langagière, sont sans doute conçus et diffusés dans ce but d'interface entre situations sociales ou professionnelles et situations de formation linguistique. Et bien que les études de V. Leclercq et A. Vicher et de C. Portefin n'apportent pas tout à fait les mêmes conclusions quant à leur utilisation par les formateurs, ils nous montrent leur prégnance dans les pratiques quotidiennes. De son côté, C. Portefin met en lumière les excès ressentis de rationalisation de l'activité du formateur que l'utilisation des référentiels semble entrainer. Ainsi, par exemple dans le référentiel RCCSP de l'ANLCI ${ }^{7}$, si l'on note bien la tentative de concilier exigence de transversalité des savoirs mobilisables au sein de l'activité professionnelle ou au sein de la vie sociale ( « calculer une quantité », « expliquer un itinéraire », « négocier un montant, un horaire ») et exigence d'adéquation des savoirs aux situations de travail ${ }^{8}$, on constate également que les savoirs sont structurés et découpés selon des catégorisations nombreuses ("pôles », « activités », « critères », « degrés de capacités professionnelles ») définissant et catégorisant une situation de travail. Ce référentiel a été conçu pour le monde professionnel comme description normative de l'activité professionnelle et non comme description de la formation (référentiel de formation), ce qui explique peut-être la technicité curriculaire dont se plaignent les formateurs. On constate encore que les savoirs prescrits à acquérir sont exclusivement fonctionnels et réfèrent à une praxis spécialisée, voire technique (« modifier ou accepter sa place dans un plan de travail collectif », « utiliser un cahier de liaison », « prendre en compte la fréquence d'évènement »). Or, lorsqu'on interroge les salariés en formation comme l'ont fait M.-H. Lachaud et D. Rehaili, ils peuvent

7. Référentiel des compétences clés en situation professionnelle, ANLCI (Agence nationale de Lutte contre l'Illettrisme).

8. Le référentiel propose d'ailleurs une grille vierge à adapter à chaque métier ou chaque poste de travail concerné par l'action de formation des salariés. 
se trouver frustrés de cette focalisation exclusive sur la langue fonctionnelle (Bachir déplore l'absence de contenus plus généraux comme l'histoire " pour savoir beaucoup de choses »). On peut, à cet effet, se demander quels effets peuvent avoir l'hyper-sélectivité et l'hypercontextualisation des contenus langagiers techniques sur le rapport aux savoirs des personnes en formation.

Les langues ne sont pas des technologies à savoir utiliser, elles ne sont pas dépossédées, «dépossédables » des individus qui les parlent ou qui s'en saisissent, elles ne sont pas des entités indépendantes des personnes et des dispositifs, elles s'y incarnent au contraire. Ainsi S. Pène (1995 : 107) écrit : «plus l'écrit est reconnu comme un moyen de représenter, de contrôler, et d'améliorer le travail d'une organisation, moins on se souvient qu'il est langage, que sa symbolique relève des personnes et non d'un "système de communication interne" ». Loin de vouloir remettre en cause le caractère parfois nécessairement spécialisé et praxique des communications humaines et donc des contenus de leur transmission, nous pensons que la grande diffusion des référentiels de compétence et la sélectivité des contenus linguistiques de formation qu'ils contiennent répond avant tout aux besoins linguistiques de l'entreprise et de la logique productive (et moins aux besoins des personnes au travail $)^{9}$ et donne à penser que les pratiques langagières se réduisent soit à des pratiques à rentabiliser, soit à des comportements observables et contrôlables de transmission d'informations ${ }^{10}$. Ainsi, la dimension relationnelle, (inter)culturelle, symbolique et non visible du langage n'est pas (ne peut pas être?) prise en compte, parce qu'elle ne se laisse peut-être pas « référencer».

En outre, tel que conçu dans des programmes ou référentiels, l'écrit standardisé doit viser l'efficacité au travail, (et est donc indice de compétence), mais en réalité il peut aussi être plus diffus, plus clandestin (notes personnelles, carnets, mails à caractère privé, billets divers, lec-

9. Ce qui amène F. Mourlhon-Dallies (2008: 312) à une mise en garde contre la confusion entre motifs de la formation et motivation pour celle-ci : « du moment que les personnes ont de bonnes raisons d'apprendre une langue, on part du principe que leur motivation est assurée ».

10. A. Duchêne (2011), S. Heller et J. Boutet (2006) montrent magistralement comment les instances politiques, institutionnelles et entrepreunariales construisent les langues comme instruments de productivité économique et comment les langues constituent «la matière première de la productivité » (Duchène, 2009 : 145). 
ture de journaux gratuits, etc.) et servir une compétence plus sociale que technique. Il y a ainsi les «écrits visibles », relevant de la prescription par l'entreprise, et les « écrits réels » (ceux de l'entreprise et/ou ceux de la personne sur son lieu de travail, informels, écrits pour soi, pour ses collègues...) (Boutet, 2008). De même, Mourlhon-Dallies (2008 : 95) aborde "l'interpénétration des espaces privés et professionnels », du fait notamment des technologies numériques, et invite à intégrer ces mutations du travail dans une démarche FLP. Il y aurait finalement un paradoxe entre la dimension opérationnelle et sélective de la langue au travail à des fins de contextualisation maximale en formation, repérable dans les discours prescrivant directement (appel d'offres) ou indirectement (référentiels) les formations et les contenus sous-contextualisés dans les pratiques réelles de formation mentionnés par $\mathrm{H}$. Adami et V. André ici-même.

\section{Langue écrite et réflexivité en formation}

Quelques-unes des contributions de ce numéro mettent en évidence le rôle de la réflexivité dans l'appropriation de la langue, réflexivité étant à entendre comme processus cognitif et communicationnel de retour sur ou de mise à distance d'une expérience (d'écriture d'un texte par exemple). Les auteurs posent la réflexivité comme un levier de transformation du rapport au savoir, indispensable dans un projet d'appropriation linguistique et important pour les adultes en formation qui, soit n'ont pas construit de rapport aux savoirs formalisés, soit ont construit des résistances, des inhibitions, des représentations figées de ceux-ci et de leur transmission.

La didactique des langues et du français a développé des travaux en ce sens pour les contextes scolaires ${ }^{11}$, mais peu de travaux se sont centrés sur les contextes de formation linguistique d'adultes à visée d'insertion, ou contexte de formation pour adultes faiblement scolarisés ${ }^{12}$. De même, tout un pan de la didactique du français s'est penché sur l'observation réfléchie de la langue, ses effets, ses intérêts et ses limites. Qu'en est-il pour les adultes en (ré)apprentissage de la langue?

11. Voir par exemple J.-C. Chabanne et D. Bucheton (2002) en français langue maternelle ou les travaux sur la conscience langagière (démarches d'éveil aux langues), sur la socialisation langagière des jeunes publics (Bigot, 2011)

12. Voir toutefois les travaux d'A. Bretegnier $(2008,2011)$. 
Ainsi, M. Niwese envisage la réflexivité dans le cadre des récits de vie en atelier d'écriture et souligne que la prise en compte du vécu dans les pratiques de formations requiert la plus grande prudence de la part des formateurs contre le risque d'instrumentalisation des parcours biographiques. Là encore, ces situations interrogent la didactique des langues et du français. Selon l'auteur, les ateliers d'écriture sont couramment proposés aux adultes en formation selon deux visées : le travail d'appropriation des textes (enjeu linguistique et didactique) et/ ou le développement personnel (enjeu psycho-affectif et psychosocial). Ce dernier but demande de réfléchir à des modalités didactiques qui, éloignées des activités très en vogue du management de l'ego, puissent répondre à des enjeux d'appropriation et à des enjeux de sécurisation linguistique, sociale et professionnelle et de transformation des personnes en formation.

De son côté, B. Balas défend la thèse que l'entrainement à l'autolangage (langage oral murmuré ou totalement intériorisé pour soi et vers soi) pourrait contribuer à faciliter le processus d'appropriation de l'écrit lorsqu'il accompagne une activité de copie de texte. L'auteur met également en avant la dimension perceptive de la langue écrite tout à fait importante dans l'acte de (ré)apprendre l'écrit. Et les descriptions de la langue, les grammaires normatives, la technologie discursive et cognitive permettant de catégoriser, ranger les pratiques langagières (à travers des référentiels, des grammaires, des tâches) fait oublier que la langue est aussi (d'abord ?) perçue, ressentie et s'établit corporellement (l'écrit passe par la bouche, l'œil, la main). C'est ainsi les aspects cognitifs et sensoriels de la langue que le formateur peut aussi privilégier pour les personnes apprenantes dans la mise en œuvre d'une situation didactique.

Enfin, à travers un dispositif de réflexion collective orale sur la langue à partir d'un texte à réécrire, F. Chnane-Davin et C. Lafarge montrent de quelle manière l'activité métalinguistique est tout à la fois une activité de conceptualisation ${ }^{13}$ (qui pourrait tout à fait rivaliser avec des ateliers de raisonnement logique), une activité d'évaluation ou de diagnostic sur un phénomène, sur un fonctionnement ou un dysfonctionnement linguistique au regard de la norme, une activité collective d'argumentation et de résolution de ce dysfonctionnement. Ces trois caractéristiques de l'activité métalinguistique peuvent constituer des compétences transversales aux situations sociales (et professionnelle a fortiori). S'ajoutent à

13. Au sens que lui donnent Besse et Porquier (1991). 
cela des enjeux identitaires, de positionnement de soi vis-à-vis du code, de la norme linguistique, de l'autre qui peuvent rejoindre des enjeux d'appropriation transversaux (apprendre à participer à une décision au travail, apprendre à prendre position dans un conflit de voisinage, etc.), moins visibles, mais jouant un rôle dans le fait d'être considéré comme compétent ou pas.

$\mathrm{Au}$ final, se dessine en creux tout le potentiel acquisitionnel du travail sur la langue elle-même ${ }^{14}$ et les effets potentiels sur le rapport aux savoirs des apprenants, quels que soient le type de dispositif et la visée formative pour les adultes en parcours d'insertion.

\section{Quelles pratiques de formation à la littéracie?}

L'étude des pratiques de formation linguistique ou de formation de base n'est pas un objet d'étude récent. Mais, comme le note V. Leclercq (2007 : 15), « la formation de base est un champ de pratiques professionnelles et sociales suscitant davantage de travaux d'ingénierie, de monographies, de comptes rendus d'expériences, de conception de supports de formation que de recherches à portée scientifique », en particulier sur le plan didactique.

Dans cette partie, deux lignes de force sont développées : les dispositifs méthodologiques adoptés par les chercheurs et certaines propriétés des pratiques de formation à la lecture-écriture.

\section{Dispositifs méthodologiques pour l'étude des pratiques de formation}

Tout d'abord, les pratiques de formation sont appréhendées dans le numéro en fonction de trois types de données : les discours déclaratifs des formateurs recueillis par entretien ou par questionnaire, les interactions de formation enregistrées et transcrites, et les discours programmatiques (référentiels). Certaines contributions s'appuient aussi explicitement sur l'observation systématique des situations (M. Niwese, C. Portefin) et/ou des acteurs (B. Balas). Cependant, les discours déclaratifs sont ici les plus sollicités, ils retiendront donc davantage notre attention. Ainsi, les discours déclaratifs de professionnels sur leur activité ouvrent l'analyse à des pratiques reconstruites et revécues pour le chercheur et dévoi-

14. Qu'il faudrait élargir aux pratiques plurilingues des personnes. 
lant une certaine épaisseur subjective de l'action didactique (Cicurel et Rivière, 2008; Rivière et Cadet, 2011). Et bien que Lahire (1998) ait expliqué la difficulté de dire le faire parce que l'action est très souvent consubstantielle à l'individu et les savoirs ou savoir-faire incorporés difficiles à verbaliser, ces « discours sur» demeurent indispensables (à condition d'expliciter leurs limites) à la compréhension de ce que font effectivement les professionnels de la formation. Néanmoins, il semble que leur puissance herméneutique serait plus grande si ce type de données, et les interprétations qu'elles permettent d'induire, s'accompagnait de l'observation et de l'analyse des pratiques langagières et interactionnelles situées. C'est ainsi que, par exemple, pour le domaine de l'éducation scolaire, les pratiques enseignantes sont quasi-systématiquement observées et analysées (Vinatier et Altet, 2008).

Ensuite, le travail important d'analyse des discours programmatiques ou de leur réception par les formateurs interroge, voire revisite, la définition même de pratique de formation. Différents auteurs du numéro l'ont mis en évidence : les pratiques actuelles de formation (linguistique) sont grandement impactées, préfigurées, et donc potentiellement standardisées, par les discours programmatiques et prescriptifs (Filliettaz, 2004).

Enfin, la focalisation unique sur le formateur exclut les effets de leurs pratiques sur les apprenants ainsi que l'étude des pratiques de formation mises en œuvre par les apprenants eux-mêmes qu'il conviendrait également de comprendre. Cette atomisation méthodologique révèle peutêtre le caractère encore éparpillé d'une didactique du français langue d'insertion.

\section{Pratiques transpositives, pratiques étayantes}

En parcourant l'ensemble des contributions, il semble que les transformations institutionnelles et organisationnelles de la formation de base amènent les formateurs à exercer une attention soutenue sur les pratiques transpositives, c'est-à-dire que leur activité est constituée d'une part importante d'ingénierie de formation nécessitant l'analyse de besoins, la sélection de contenus, la planification et la mise en place effective de la formation et suscite, lors de ces différentes phases, un travail important de sélection, de traduction et de transposition des savoirs à enseigner. Ainsi les formations linguistiques dans le cadre du CAI ont comme finalité l'obtention du DILF. Les formateurs recourent alors au Référentiel DILF-Niveau A1. 1 pour le français et y sélectionnent des propositions de contenus ; ils élaborent les supports et les activités correspondant aux 
épreuves certifiantes du DILF. Pour la formation linguistique en situation de travail, la transposition s'articule selon deux modalités croisées : transposition des pratiques langagières et interactionnelles observées in situ et transposition des savoirs listés dans les Référentiels métiers. Il serait tout à fait intéressant à cet égard d'étudier toutes les traces langagières de cette chaine transpositive et leurs transformations aux différentes phases de l'activité du formateur afin de mettre au jour ces pratiques transpositives et leur complexité ${ }^{15}$.

Une autre propriété des pratiques de formation repérables au fil des contributions (Balas, Niwese, Chnane-Davin et Lafarge) concerne les pratiques étayantes et collectives sur la langue. Elles concernent plus spécifiquement la phase de face-à-face pédagogique et consistent à guider les apprenants dans le processus d'incorporation de nouvelles normes (à l'écrit ou à l'oral) par la gestion de la dynamique de la parole collective, voire de la parole auto-centrée. Ces pratiques de formation étayantes cherchent à provoquer le changement et la transformation des représentations de l'écrit chez les apprenants, par le guidage du processus de construction de nouvelles normes de l'écrit et concourent à rendre moins étrangère la langue apprise ou réapprise (Trevise, 1992). En acquisition des langues, l'activité métalinguistique, ses caractéristiques, ses effets sont bien décrits. En particulier, les études menées sur la conscientisation de la langue en situation informelle et exolingue de communication (Vasseur, 1996) pourraient être utilement remobilisés si on voulait s'atteler à penser les processus d'appropriation comme un continuum entre situations non guidées de la vie sociale et professionnelle et situations guidées de formation.

\section{Quelques propositions finales prospectives}

La richesse des contributions de ce numéro nous amène à synthétiser quelques pistes de recherche en didactique des langues et du français qui permettraient d'intensifier le processus de documentation et de construction d'intelligibilité du domaine de la formation linguistique des adultes

15. Nous partons du principe que la formation linguistique pour adultes manque de légitimité sociale et institutionnelle (Leclercq, 2007). Des efforts de recherche sur ce champ en didactique des langues, joints à des efforts d'articulation avec les recherches en sciences de la formation, pourraient notamment contribuer à accroitre cette légitimité. 
en insertion. Les pratiques de formation sont centrales. En effet, « quel que soit le paradigme de pensée, les "pratiques" comme objets d'analyse concernent la totalité de l'individu, en tant que sujet, dans ses relations avec l'environnement dans les différents contextes de sa vie personnelle, professionnelle et sociale ( Marcel, Olry et al., 2002).

Premièrement, un travail impérieux de description et de typologisation des documents écrits du travail permettrait en bout de chaine didactique que les formateurs soient mieux armés pour entrer dans ces écrits parfois complexes et pour les didactiser.

Ensuite, les discours programmatiques, leur utilisation et leur appropriation ou non par les acteurs de la formation, et en premier lieu les formateurs, pourraient constituer aussi un programme fort riche de recherche parce qu'il nous apprendrait sur les conceptions de la langue, de l'acte de lire et d'écrire que peuvent avoir les auteurs de ces outils.

Enfin, l'étude située des pratiques de formation, aux différents stades de leur déploiement et selon différentes échelles de temps, ainsi que l'étude de leur effets, sont à poursuivre et intensifier. Si le processus de professionnalisation des acteurs de la formation est engagé et intensifié, il ne peut se faire sans connaissances préalables organisées et systématisées des pratiques réelles, de leurs invariants et de leur variété.

\section{RÉFÉRENCES BIBLIOGRAPHIQUES}

Besse H. et Porquier L. (1991): Grammaires et didactique des langues, Paris, Didier.

Bigot V. (2011) : «Co-écriture et socialisation langagière des élèves moins experts en français. L'ex-pair-tise du locuteur natif sollicitée », dans C. Le Cunff et M.-A. Hugon (éds), Interactions dans le groupe et apprentissages, Publications de l'université Paris Ouest-Nanterre-La Défense.

BRETEGNIER A. (2008) : «Interroger le sens et les enjeux des projets d'appropriation du français langue d'insertion : ouverture d'un champ de recherches interventions », Glottopol, $\mathrm{n}^{\circ}$ 11, Rouen, p. 70-80.

BRETEGNIER A. (2011) : Formation linguistique en contextes d'insertion, Berne, Peter Lang.

Boutet J. (2001) : « La part langagière du travail : bilan et évolutions », Langage et société, n 98, Paris, Éditions de la MSH, p. 17-42.

Boutet J. (2008) : La vie verbale au travail. Des manufactures aux centres d'appel, Toulouse, Octarès Éditions. 
Chabanne J.-C. et Bucheton D. (2002) : Parler et écrire pour penser, apprendre et se construire. L'écrit et l'oral réflexifs, Paris, Presses universitaires de France.

Cicurel F. et Riviere V. (2008) : « De l'interaction en classe à l'action revécue : le clair-obscur de l'action enseignante », dans L. Filliettaz et M.-L. Schubauer-Leoni (éds), Processus interactionnels et situations éducatives, Bruxelles, De Boeck, p. 255-273.

DuChÊNE A. (2009) : «Formé-e pour servir! la part langagière de la formation professionnelle dans la nouvelle économie », Bulletin suisse de linguistique appliquée, $\mathrm{n}^{\circ}$ 90, p. 125-147.

DuchÊNE A. (2011) : « Néolibéralisme, inégalités sociales et plurilinguisme : l'exploitation des ressources langagières et des locuteurs », Langage et société, $\mathrm{n}^{\circ}$ 136, Paris, Éditions de la MSH, p. 81-108.

Filliettaz L. (2004) : «Une sémiologie de l'agir au service de l'analyse des textes procéduraux », Cahiers de la section des sciences de l'éducation, $\mathrm{n}^{\circ}$ 103, Genève, p. 147-184.

Fraenkel B. et Borzeix A. (2001) : Langage et Travail, Communication, Cognition, Action, Paris, Éditions du CNRS.

Grossman F. (1998) : «Le texte écrit : un empêcheur de dialoguer en rond? », dans F. Grossman (dir.), Pratiques langagières et didactique de l'écrit, Grenoble, Ivel-Lidilem.

Heller M. et Boutet J. (2006) : « Vers de nouvelles formes de pouvoir langagier? Langue(s) et identités dans la nouvelle économie », Langage et société, $\mathrm{n}^{\circ} 118$, Paris, Éditions de la MSH, p. 5-16.

Lacoste M. (1995) : «Parole, action, situation », dans J. Boutet (dir.), Paroles au travail, Paris, L'Harmattan, p. 23-44.

LAHIRE B. (1998) : «Logiques pratiques : le "faire" et le "dire sur le faire" », Recherche et Formation, $\mathrm{n}^{\circ}$ 27, Paris, INRP, p. 15-28.

LECLERCQ V. (2007) : « La formation de base : publics, dispositifs, pratiques », Savoirs, $\mathrm{n}^{\circ}$ 14, p. 8-55, Paris, L'Harmattan.

Marcel J.-F., Olry P., Rothier-Bautzer E., Sonntag M. (2002) : « Les pratiques comme objet d'analyse », Revue française de pédagogie, $\mathrm{n}^{\circ} 138$, Lyon, INRP, p. 135-170.

Mourlhon-Dallies F. (2008) : Enseigner une langue à des fins professionnelles, Paris, Didier.

Pène S. (1995) : « Traces de mains sur des écrits gris », dans J. Boutet (dir.), Paroles au travail, Paris, L'Harmattan, p. 105-122.

Rivière V. et CADET L. (2011) : «Mise en perspective des savoirs professionnels dans des discours de retour d'expérience. Étude contrastée de deux enseignantes de français langue étrangère ", LIDIL, $\mathrm{n}^{\circ} 43$, Grenoble, ELLUG, p. 41-56. 
Rispail M. et Blanchet P. (2011) : « Principes transversaux pour une sociodidactique dite "de terrain" », dans P. Blanchet et P. Chardenet (dir.), Guide pour la recherche en didactique des langues, Paris, EAC.

Trevise A. (1992) : "La gestion cognitive de l'étrangeté dans l'acquisition d'une langue étrangère », AILE, $\mathrm{n}^{\circ} 1$, Saint-Denis, université de Paris VIII, p. 87-106.

VASSEUR M.-T. (1996) : «Les activités réflexives en situation de communication exolingue : réflexions sur quinze ans de recherche », AILE, $\mathrm{n}^{\circ} 8$, Saint-Denis, université de Paris VIII, p. 57-87.

Vinatier I. et Altet M. (2008) : Analyser et comprendre la pratique enseignante, Rennes, Presses universitaires de Rennes. 